\title{
The medical and legal ramifications of women with AIDS
}

The World Health Organization designated Dec 1, 1990 as "Women and AIDS" Day. This third annual World AIDS Day featured special events designed to emphasize the impact of AIDS on women. Of the 8 million persons with AIDS, one third are women. Intravenous drug use, particularly needle-sharing among users, continues to be a major route for HIV transmission in women. The Centers for Disease Control (CDC) reports that diagnosed AIDS cases increased $29 \%$ in women from 1988 to 1989 . During that same period, the incidence of AIDS in men rose $18 \%$. If the current trend continues, AIDS will be among the five leading causes of death in women aged 15 to 44 years this year, according to $\mathrm{CDC}$ estimates.

Of all AIDS cases in women, $85 \%$ occur among women in their childbearing years, namely between the ages of 15 and 44 years. Thus, AIDS poses not only a health threat to women, but also to their unborn children. For example, in a recent Florida study, 1 out of every 220 mothers giving birth tested positive for the HIV. Thus, potentially 1 out of every 220 infants in Florida alone may be infected with the HIV. Even if the number of HIVinfected mothers is lower in the other 49 states, the potential health implications are enormous. Coupled with these sheer numbers is the estimated median 11-year HIV incubation period.

With such a large pool of women-and their children - at risk for HIV infection, what role do physicians play in this scenario? We must first realize that many sexually active women are unaware of their risk of contracting the HIV. Our advice and counseling with respect to sexual matters are critical. Other than in a long-term, monogamous relationship, sexual activity is potentially hazardous. We physicians have an obligation to inform and counsel patients accordingly.
But what responsibility do we have to notify the sexual or needle-sharing partners of our HIV-infected patients? Beginning on page 46, Scott H. Isaacman, DO, JD, and Michael L. Closen, JD, write that physicians who care for HIV-infected patients have a duty to assure that at-risk third parties are notified of their respective risks of infection. The authors write that the $\mathrm{CDC}$ guidelines recommend the following: "If [HIV-infected persons] are unwilling to notify their partners or if it cannot be assured that their partners will seek counseling, physicians or health department personnel should use confidential procedures to assure that the partners are notified."

The authors conclude, "A physician who fails to disclose a patient's HIV infection to identified or reasonably identifiable sexual or needle-sharing partners (or both) may become embroiled in a negligence claim based on failure to warn."

Certainly we have a legal obligation to warn third parties of their partners' infection. However, we have a medical and perhaps even a moral obligation to not only counsel our patients but their partners as well.

THOMAS WESLEY ALLEN, DO Editor in Chief

\section{An example of putting our assumptions to the test}

Much of what we assume to be true in medicine has not been tested; objective evidence of cause and effect is often lacking. Case in point is the relationship between unlevelness of the sacral base, scoliosis, and back pain. Osteopathic physicians have long assumed a causeand-effect relationship. However, as Robert E. (continued on page 28) 
Irvin, DO, points out in his article beginning on page 37, a lack of objective evidence exists in this area. Specifically, no objective evidence has previously been reported supporting the causal relationship between the unlevel sacrum and scoliosis and the potential benefit of lift therapy in alleviating mild cases of lumbar scoliosis.

Dr Irvin's study of 51 adults with mild scoliosis examines the effect of lift therapy in this patient population. Patients in his study had a sacral base unlevelness ranging from 2 to $17 \mathrm{~mm}$ and a lateral bend in the lumbar spine ranging from 2 to 19 degrees. An anteroposterior x-ray film of the lumbar spine and pelvis was taken for each subject before and after treatment. Patients wore a lift composed of cork inside their shoe. Every 2 weeks, the heel lift was augmented by $1.6 \mathrm{~mm}$. At the same time, study participants were examined for restricted normal freedom of motion of the soft tissues and joints. This treatment regimen lasted for 44 weeks.

Results were significant. The sacral base unlevelness was reduced from an initial mean of $6.7 \pm 1.0 \mathrm{~mm}$ to a mean of $2.6 \pm 0.4 \mathrm{~mm}$ following lift therapy. Likewise, after treatment, the angle of lateral bend among these patients was reduced to a mean of $5.3 \pm 0.8$ degrees from a pretreatment average of $7.5 \mathrm{de}$ grees. Although some patients in Dr Irvin's study experienced discomfort as the final $20 \%$ of unlevelness was corrected, their discomfort (myalgia, slight nausea, and malaise) lasted only 1 to 7 days and was more common among patients who initially had a greater degree of scoliosis. Overall, Dr Irvin's results show that lift therapy can reduce mild scoliosis in adults by approximately one third.

Dr Irvin's valuable work is but one example of the type of investigations in progress throughout our profession. This study exemplifies an objective description of what we DOs have assumed for years. The JAOA welcomes such documented objective observation and description.
THOMAS WESLEY ALLEN, DO Editor in Chief 


\section{BUMEX:}

bumetanide/Roche

$0.5-\mathrm{mg}, 1-\mathrm{mg}$ and $2 \cdot \mathrm{mg}$ scored tablets

2-ml ampuls $(0.25 \mathrm{mg} / \mathrm{ml})$ and

$2 \cdot \mathrm{ml}, 4-\mathrm{ml}$ and $10 \mathrm{ml}$ vials $(0.25 \mathrm{mg} / \mathrm{ml})$

Before prescribing, please consult complete product information, a summary of which follows:

WARNING: Bumex (bumetanide/Roche) is a potent diuretic which if given in excessive amounts, can lead to profound diuresis with water and electrolyte depietion. Therefore, amounts, can lead to prision is required, and dose and dosage schedule have to be adjusted to the individual patient's needs. (See under DOSAGE AND ADMINISTRATION in complete product information.)

INDICATIONS AND USAGE: Edema associated with congestive heart failure, hepatic and renal disease, including the nephrotic syndrome

Almost equal diuretic response occurs after oral and parenteral administration of Bumex. If impaired gastrointestinal absorption is suspected or oral administration is not practical, Bumex should be given by the intramuscular or intravenous route.

Successful treatment with Bumex following instances of allergic reactions to furosemide suggests lack of cross-sensitivity

CONTRAINDICATIONS: Anuria. Hypersensitivity and in patients in hepatic coma or in states of severe electrolyte depletion. Although Bumex can be used to induce diuresis in renal insufficiency. severe electrolyte depletion. Aithough Bumex can be used to induce diuresis in renal insufficiency any marked increase in blood urea nitrogen or creatinine, or the development of oliguria during WARNINGS: Dose should be adjusted to patient's needs. Excessive doses or too frequent

WARNINGS: Dose should be adjusted to patient's needs. Excessive doses or too frequent

administration can lead to profound water loss, electrolye depietion, dehydration, reduction in blood volume and circulatory collapse

particularly in elderly patients.

Prevention of hypokalemia requires particular attention in patients receiving digitalis and diuretics for congestive heart failure, hepatic cirrhosis and ascites, states of aldosterone excess with norma renal function, potassium-losing nephropathy, certain diarrheal states, or other states where hypokalemia is thought to represent particular added risks to the patients.

In patients with hepatic cirrhosis and ascites, sudden aiterations of electrolyte balance may precipitate hepatic encephalopathy and coma. Treatment in such patients is best initiated in the hospital with small doses and careful monitoring of the patient's clinical status and electrolyte balance. Supplemental potassium and/or spironolactone may prevent hypokalemia and metabolic alkalosis in these patients.

In cats, dogs and guinea pigs. Bumex has been shown to produce ototoxicity. Since Bumex is abou 40 to 60 times as potent as furosemide, it is anticipated that blood leveis necessary to produce ototoxicity will rarely be achieved. The potential for ototoxicity increases with intravenous therapy. especially at high doses.

Patients allergic to sulfonamides may show hypersensitivity to Bumex

PRECAUTIONS: Measure serum potassium periodically and add potassium supplements or

potassium-sparino diuretics, if necessary. Periodic determinations of other electrolytes are advised in patients treated with high doses or for prolonged periods, particularly in those on low salt diets.

Hyperuricemia may occur. Reversible elevations of the BUN and creatinine may occur, especially with dehydration and in patients with renal insufficiency Bumex may increase urinary calcium

excretion

Possibility of effect on glucose metabolism exists. Periodic determinations of blood sugar should be done, particularly in patients with diabetes or suspected latent diabetes

Patients should be observed regularly for possible occurrence of blood dyscrasias, liver damage or idiosyncratic reactions.

Especially in presence of impaired renal function, use of parenterally administered Bumex should be avoided in patients to whom aminoglycoside antibiotics are also being given, except in lifethreatening conditions.

Drugs with nephrotoxic potential and bumetanide should not be administered simultaneously

Since lithium reduces renal clearance and adds a high risk of lithium toxicity, it should not be given with diuretics.

Probenecid should not be administered concurrently with Bumex

Concurrent therapy with indomethacin not recommended.

Bumex may potentiate the effects of antihypertensive drugs, necessitating reduction in dosage.

Interaction studies in humans have shown no effect on digoxin blood levels

Interaction studies in humans have shown Bumex to have no effect on wartarin metabolism or on plasma prothrombin activity.

Pregnancy: Bumex should be given to a pregnant woman only if the potential benefit justifies the potential risk to the fetus.

Bumetanide may be excreted in breast milk.

Pediatric use: Safety and effectiveness below age 18 not established.

ADVERSE REACTIONS: Muscle cramps, diziness, hypotension, headache and nausea, and

encephalopathy (in patients with preexisting liver disease).

Less frequent clinical adverse reactions are weakness, impaired hearing, rash, pruritus, hives, electrocardiogram changes, abdominal pain, arthritic pain, musculoskeletal pain and vomiting. Other clinical adverse reactions are vertigo, chest pain, ear discomfort. fatique, dehydration, sweating, hyperventilation, dry mouth, upset stomach, renal failure, asterixis, itching, nipple tenderness, diarmea, premature ejaculation and difficulty maintaining an erection.

Laboratory abnormalities reported are hyperuricemia, azotemia, hyperglycemia, increased serum creatinine, hypochloremia, hypokaiemia, hyponatremia and variations in $\mathrm{CO}_{2}$ content, bicarbona phosphorus and calcium. Although manifestations of the phar

Also reported have been thrombocytopenia, deviations in hemoglobin, prothrombin time. hematocrit. WBC and differential counts. There have been rare spontaneous reports of thrombocytopenia from postmarketing experience.

Diuresis induced by Bumex may also rarely be accompanied by changes in $\mathrm{LDH}$, total serum bilirubin, serum proteins, SGOI, SGPT, alkaline phosphatase, cholesterol, creatinine clearance. Increases in urinary glucose and urinary protein have also been seen

DOSAGE AND ADMINISTRATION:

Oral Administration: The usual total daily dosage is $0.5102 .0 \mathrm{mg}$ and in most patients is given as a single dose.

Parenteral Administration: Administer to patients (IV or IM) with GI absorption problem or who can not take oral. The usual initial dose is 0.5 to $1 \mathrm{mg}$ given over 1 to 2 minutes. If insufficient response, a second or third dose may be given at 2 to 3 hour intervals up to a maximum of $10 \mathrm{mg}$ a day. HOW SUPPLIED: ablets, $0.5 \mathrm{mg}$ (light green), $1 \mathrm{mg}$ (yellow) and $2 \mathrm{mg}$ (peach), bottles of 100 and 500: Tel-E Dose* cartons of 100. Imprint on tablets: $0.5 \mathrm{mg}-$ ROCHE BUMEX 0.5:1 mg - ROCHE BUMEX 1;2 mg - ROCHE BUMEX 2 Ampuls, $2 \mathrm{ml}, 0.25 \mathrm{mg} / \mathrm{ml}$, boxes of ten Vials, $2 \mathrm{ml}, 4 \mathrm{ml}$ and $10 \mathrm{ml}, 0.25 \mathrm{mg} / \mathrm{ml}$, boxes of ten.

Store all tablets, vials and ampuls at $59^{\circ}$ t0 $86^{\circ}$.

\section{Roche Laboratories}

\section{a division of Hoffmann-La Roche Inc}

340 Kingsiand Street. Nutley, New Jersey 07110-1199

Working today for a healthier tomorrow.

\section{We Wrote the Books on Patient Medication Education}

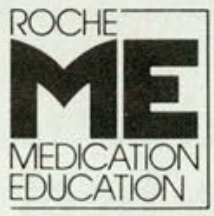

\section{Now we've written them in Spanish}

In 1978, Hoffmann-La Roche pioneered pharmaceutical patient information with the introduction of the Roche Medication Education (ME) program. We stand ready to meet the growing need for patient medication education in your practice. We invite you to take part in this valuable patient information program.

By simply checking the boxes below and mailing the coupon to Roche Medication Education, Roche Laboratories, a division of Hoffmann-La Roche Inc., Nutley, New Jersey 07110-1199, you may obtain a complimentary supply of the booklets providing specific information on Roche branded products listed below.

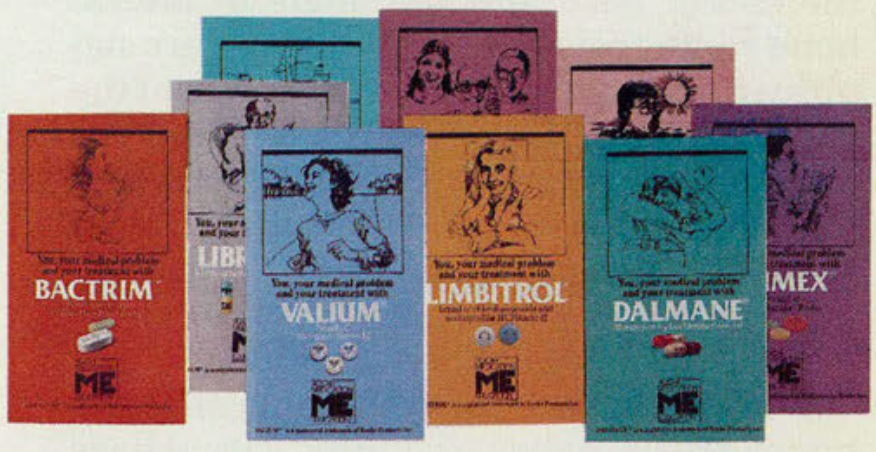

\section{Roche Product Books}

English Spanish version version

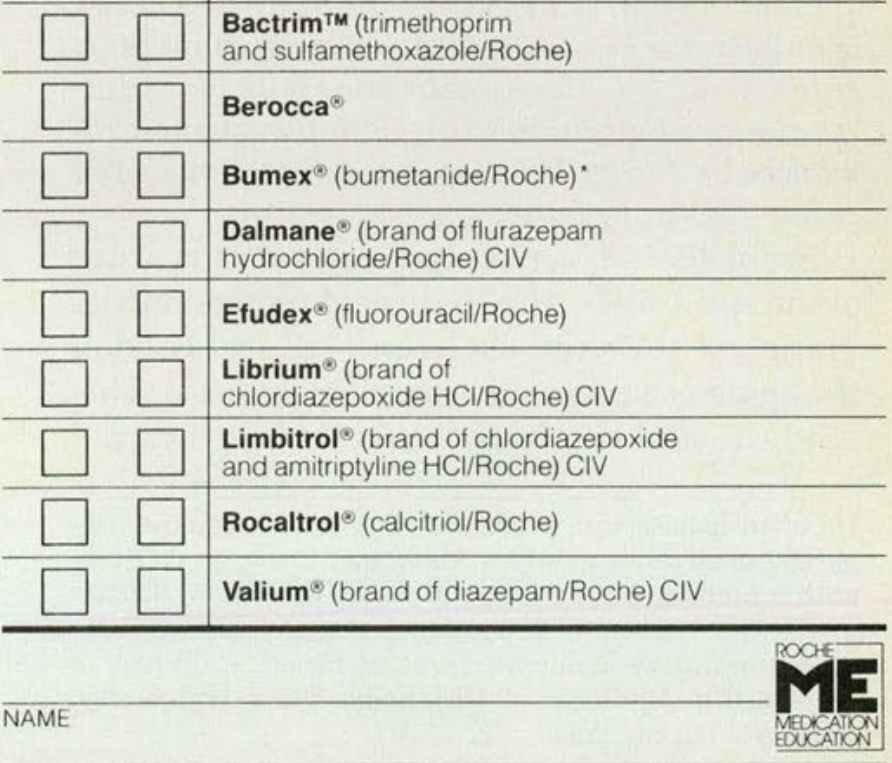


Ha|c|ロ Tablets (triazolam) @

INDICATIONS AND USAGE: HALCION Tablets are indicated in the short-term management of insomnia characterized by difficulty in falling asleep, frequent nocturnal awakenings, and/or early morning awakenings. It is recommended that HALCION not be prescribed in quantities exceeding a one-month supply.

CONTRAINDICATIONS: Patients with known hypersensitivity to this drug or other

benzodiazepines.

HALCION is contraindicated in pregnant women due to potential fetal damage. Patients likely to become pregnant while receiving HALCION should be warned of the potential risk to the fetus.

WARNINGS: Overdosage may occur at four times the maximum recommended therapeutic dose. Patients should be cautioned not to exceed prescribed dosage.

Because of its depressant CNS effects, patients should be cautioned against engaging in hazardous occupations requiring complete mental alertness and also about the simultaneous ingestion of alcohol and other CNS depressant drugs.

Anterograde amnesia and paradoxical reactions have been reported with HALCION and some other benzodiazepines.

PRECAUTIONS: General: In elderly and/or debilitated patients, treatment should be initiated at $0.125 \mathrm{mg}$ to decrease the possibility of development of oversedation, dizziness, or impaired coordination. Some side effects, including drowsiness, dizziness, lightheadedness, and amnesia, appear to be dose related.

Some evidence suggests that confusion, bizarre or abnormal behavior, agitation, and hallucinations may also be dose related, but this evidence is inconclusive. It is recommended that therapy be initiated at the lowest effective dose. Caution should be exercised in patients with signs or symptoms of depression which could be intensified by hypnotic drugs. Suicidal tendencies and intentional overdosage is more common in these patients The usual precautions should be observed in patients with impaired renal or hepatic function and chronic pulmonary insufficiency. Information for Patients: Alert patients about: (a) consumption of alcohol and drugs, (b) possible fetal abnormalities, (c) operating machinery or driving, (d) not increasing prescribed dosage, (e) possible worsening of sleep atter discontinuing HALCION. Laboratory Tests: Not ordinarily required in otherwise healthy patients. Drug Interactions: Additive CNS depressant effects with other psychotropics, anticonvulsants, antihistaminics, ethanol, and other CNS depressants. Pharmacokinetic interactions of benzodiazepines with other drugs have been reported, e.g., coadministration with either cimetidine or erythromycin approximately doubled the elimination half-life and plasma levels of triazolam, hence increased clinical observation and consideration of dosage reduction may be appropriate. Carcinogenesis, Mutagenesis, Impairment of Fertility: No evidence of carcinogenic potential was observed in mice during a 24-month study with HALCION in doses up to 4000 times the human dose. Pregnancy: Benzodiazepines may cause fetal damage if administered during pregnancy. The child born of a mother who is on benzodiazepines may be at some risk for withdrawal symptoms and neonatal flaccidity during the postnatal period. Nursing Mothers: Administration to nursing mothers is not recommended. Pediatric Use: Safety and efficacy in children below the age of 18 have not been established.

ADVERSE REACTIONS: During placebo-controlled clinical studies in which 1003 patients received HALCION Tablets, the most troublesome side effects were extensions of the pharmacologic activity of HALCION, e.g., drowsiness, dizziness, or lightheadedness.

\begin{tabular}{lcc} 
& HALCION & Placebo \\
\hline Number of Patients & 1003 & 997 \\
\hline \% of Patients Reporting: & & \\
Central Nervous System & 14.0 & 6.4 \\
Drowsiness & 9.7 & 8.4 \\
Headache & 7.8 & 3.1 \\
Dizziness & 5.2 & 4.5 \\
Nervousness & 4.9 & 0.9 \\
Lightheadedness & 4.6 & 0.8 \\
Coordination Disorder/Ataxia & & \\
Gastrointestinal & 4.6 & 3.7 \\
Nausea/Vomiting & &
\end{tabular}

In addition, the following adverse events have been reported less frequently (i.e., 0.9-0.5\%): euphoria, tachycardia, tiredness, confusional states/memory impairment, cramps/pain, depression, visual disturbances.

Rare (i.e., less than $0.5 \%$ ) adverse reactions included constipation, taste alterations, diarrhea, dry mouth, dermatitis/allergy, dreaming/nichtmares, insomnia, paresthesia, tinnitus, dysesthesia, weakness, congestion, death from hepatic failure in a patient also receiving diuretic drugs.

The following adverse events have been reported in association with the use of HALCION and other benzodiazepines: Amnestic symptoms, confusional states, dystonia, anorexia, fatigue, sedation, slurred speech, jaundice, pruritus, dysarthria, changes in libido, menstrual irregularities, incontinence and urinary retention.

Other events reported include: Paradoxical reactions such as stimulation, agitation, increased muscle spasticity, sleep disturbances, hallucinations, aggressiveness, falling. somnambulism, inappropriate behavior, and other adverse behavioral effects. Should these occur, use of the drug should be discontinued.

No laboratory changes were considered to be of physiological significance.

When treatment is protracted, periodic blood counts, urinalysis and blood chemistry analyses are advisable.

Minor changes in EEG patterns, usually low-voltage fast activity have been observed in patients during HALCION therapy and are of no known significance.

DRUG ABUSE AND DEPENDENCE: Controlled Substance:HALCION Tablets are a Controlled Substance in Schedule IV. Abuse and Dependence: Withdrawal symptoms have occurred following abrupt discontinuance of benzodiazepines. Patients with a history of seizures are at particular risk. Addiction-prone patients should be closely monitored. Repeat prescriptions should be limited to those under medical supervision.

OVERDOSAGE: Because of the potency of triazolam, overdosage may occur at $2 \mathrm{mg}$, four times the maximum recommended therapeutic dose $(0.5 \mathrm{mg})$. Manifestations of overdosage include somnolence, confusion, impaired coordination, slurred speech, and ultimately, coma. Respiration, pulse, and blood pressure should be monitored and supported by general measure when necessary. Immediate gastric lavage should be performed. Multiple agents may have been ingested.

Store at controlled room temperature $15^{\circ}-30^{\circ} \mathrm{C}\left(59^{\circ}-86^{\circ} \mathrm{F}\right)$

Caution: Federal law prohibits dispensing without prescription.
MOVING?

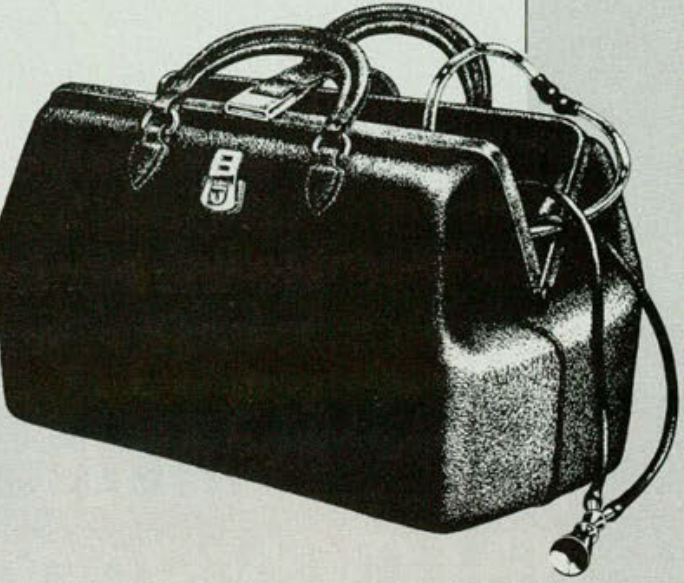

Don't forget The JA0A

Attach your current mailing label, print your new address in the space provided, and mail this form to:

Circulation Department

American Osteopathic

Association

142 E. Ontario St.

Chicago, IL 60611

New Address:

Name

AOA Number

Address

City/State/Zip

\section{AFFIX LABEL HERE}

Please allow 4-6 weeks for delivery. 


\section{In Bronchitis and Otitis Media*}
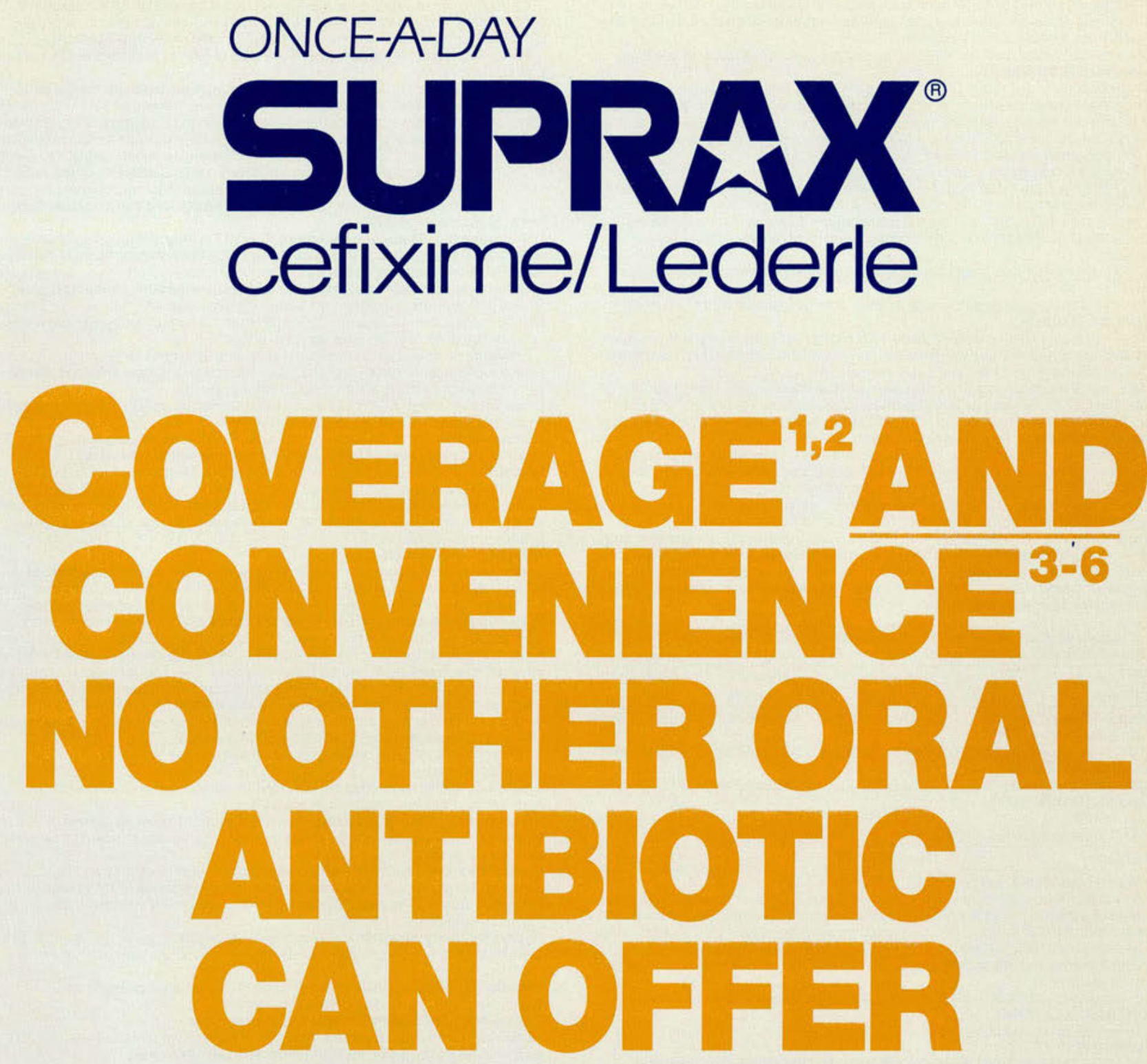

- Broad in vitro ${ }^{\dagger}$ spectrum ${ }^{1,2}$

- Highly active against key respiratory pathogens ${ }^{\text {t7-9 }}$

- Proven effective in clinical trials,10-13

* Due to susceptible organisms.

${ }^{\dagger}$ Although a useful guide, in vitro activity may not correlate with clinical response.

SUPRAX may be administered as a single dose, once a day, or in divided doses bid if preferred.

References: 1. Data on file, Lederle Laboratories, Pearl River, NY. 2. Neu HC: Beta-lactamase stability of cefixime. Internal Medicine 1990;11:57-71. 3. A national study of consumer experience with antibiotic therapy. Princeton, NJ, The Gallup Organization, Inc., July 1989. Data on file, Lederle Laboratories, Pearl River, NY. 4. Cockburn J, Gibberd RW, Reid AL, et al: Determinants of non-compliance with short term antibiotic regimens. Br Med J 1987;295:814-818. 5. Hussar DA: Importance of patient compliance in effective antimicrobial therapy. Pediatr Infect Dis J 1987:6:971-975. 6. Greenberg RN: Overview of patient compliance with medication dosing: A literature review. Clin Ther 1984;6:592-599. 7. Neu HC, Chin N-X, Labthavikul P: Comparative in vitro activity and B-lactamase stability of FR 17027, a new orally active cephalosporin. Antimicrob Agents Chemother 1984,26:174-180. 8. Neu HC: In vitro activity of a new broad spectrum, beta-lactamase-stable oral cephalosporin, cefixime. Pediatr Infect Dis J1987;6:958-962. 9. Sanders CC: B-lactamase stability and in vitro activity of oral cephalosporins against strains possessing well-characterized mechanisms of resistance. Antimicrob Agents Chemother 1989;33:1313-1317. 10. Pichichero ME. Cefixime multicenter national ottis media study, in The Contemporary Treatment of Othis Media 1990. Lederle Laboratories. Data on file, Lederle Laboratories, Peari River, NY. 11. Howie VM, Owen MJ: Bacteriologic and clinical efficacy of cefixime compared with amoxicilin in acute otitis media. Pediatr Infect Dis J 1987; 6:989-991. 12. Verghese A, Roberson D, Kalbfleisch JH, et al: Randomized comparative study of cefixime versus cephalexin in acute bacterial exacerbations of chronic bronchitis. Antimicrob Agents Chemother 1990;34:1041-1044. 13. Kiani R, Johnson D, Nelson B: Comparative, multicenter studies of cefixime and amoxicillin in the treatment of respiratory tract infections. Am J Med 1988;85:6-13.

Please see brief summary of Prescribing Information on adjacent page. 
SUPRAX ${ }^{*}$ cefixime/Lederle

BRIEF SUMMARY. Please see package insert for full Prescribing Information INDICATIONS ANDUSAGE

Otitis Media caused by Haemophilus influenzae (beta-lactamase positive and negative strains), Moraxella (Branhamella) catarrhalis (most of which are beta-lactamase positive), and Streptococcus pyogenes.

Note: For information on otitis media caused by Streptococcus pneumoniae, see CLINICAL STUDIES section.

Acute Bronchitis and Acute Exacerbations of Chronic Bronchitis caused by S pneu moniae and $H$ influenzae (beta-lactamase positive and negative strains)

Perform culture and susceptibility studies to determine causative organism and its susceptibility to SUPRAX. Therapy may begin while waiting for study results and may be adjusted when results are known.

Pharyngitis and Tonsillitis caused by S pyogenes

Note: Penicillin is the usual drug of choice in the treatment of $S$ pyogenes infections,

including the prophylaxis of rheumatic fever. SUPRAX is generally effective in

the eradication of $S$ pyogenes from the nasopharynx; however, data establishing

the efficacy of SUPRAX in the subsequent prevention of rheumatic fever are not available.

Uncomplicated Urinary Tract Infections caused by Escherichia coli and Proteus mirabilis.

'Efficacy for this organism was studied in fewer than ten patients with otitis media. CLINICAL STUDIES

In clinical trials of otitis media in nearly 400 children between the ages of 6 months and 10 years, S pneumoniae was isolated from $47 \%$ of the patients, $H$ influenzae from $34 \%, B$ catarrhalis from $15 \%$, and S pyogenes from $4 \%$

The overall response rate of $S$ pneumoniae to cefixime was approximately $10 \%$ lower and that of $H$ influenzae or B catarrhalis approximately $7 \%$ higher $(12 \%$ when beta-lactamase positive strains of $H$ influenzae are included) than the response rates of these organisms to the active control drugs.

In these studies, patients were randomized and treated with either cefixime at dose regimens of $4 \mathrm{mg} / \mathrm{kg}$ bid or $8 \mathrm{mg} / \mathrm{kg}$ qd, or with a standard antibiotic regimen. Sixtynine to $70 \%$ of the patients in each group had resolution of signs and symptoms of otitis media when evaluated two to four weeks posttreatment, but persistent effusion was found in $15 \%$ of the patients. When evaluated at the completion of therapy, $17 \%$ of patients receiving cefixime and $14 \%$ of patients receiving effective comparative drugs (18\% including those patients who had $H$ influenzae resistant to the control drug and who received the control antibiotic) were considered to be treatment failures. By the two-to four-week follow-up, a total of $30 \%$ to $31 \%$ of patients had evidence of either treatment failure or recurrent disease.

Bacteriological Outcome of Otitis Media at Two- to Four-Weeks Posttherapy Based on Repeat Middle Ear Fluid Culture or Extrapolation from Clinical Outcome

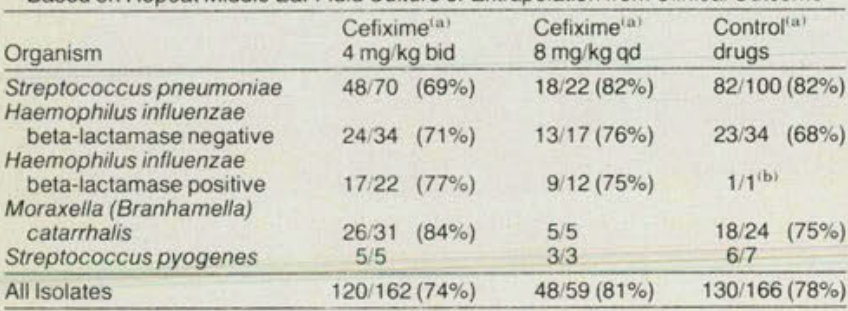

(a) Number eradicated/number isolated

An additional 20 beta-lactamase positive strains of $H$ influenzae were isolated, but were excluded from this analysis because they were resistant to the control antibiotic. In 19 of these the clinical course could be assessed, and a favorable outcome occurred in 10. When these cases are included in the overall bacteriological evalua tion of therapy with the control drugs, $140 / 185(76 \%)$ of pathogens were considered to be eradicated

Tablets should not be substituted for suspension when treating otitis media CONTRAINDICATIONS

Known allergy to cephalosporins

WARNINGS

BEFORE THERAPY WITH SUPRAX IS INSTITUTED, CAREFUL INQUIRY

SHOULD BE MADE TO DETERMINE WHETHER THE PATIENT HAS HAD PREVI OUS HYPERSENSITIVITY REACTIONS TO CEPHALOSPORINS, PENICILLINS OR OTHER DRUGS. IF THIS PRODUCT IS TO BE GIVEN TO PENICILLINSENSITIVE PATIENTS, CAUTION SHOULD BE EXERCISED BECAUSE CROSS. HYPERSENSITIVITY AMONG BETA-LACTAM ANTIBIOTICS HAS BEEN CLEARLY DOCUMENTED AND MAY OCCUR IN UP TO $10 \%$ OF PATIENTS WITH A HISTORY OF PENICILLIN ALLERGY. IF AN ALLERGIC REACTION TO SUPRAX OCCURS DISCONTINUE THE DRUG. SERIOUS, ACUTE HYPERSENSITIVITY REACTIONS MAY REQUIRE TREATMENT WITH EPINEPHRINE AND OTHER EMERGENCY MEASURES, INCLUDING OXYGEN, INTRAVENOUS FLUIDS, INTRAVENOUS ANTIHISTAMINES, CORTICOSTEROIDS, PRESSOR AMINES, AND AIRWAY MANAGEMENT, AS CLINICALLY INDICATED.

Administer cautiously to allergic patients.

Treatment with broad-spectrum antibiotics alters the normal flora of the colon and may permit overgrowth of clostridia. Studies indicate that a toxin produced by Clostridium difficile is a primary cause of severe antibiotic-associated diarrhea including pseudomembranous colitis. Pseudomembranous colitis has been reported with the use of SUPRAX and other broad-spectrum antibiotics (including macrolides, semisynthetic penicillins, and cephalosporins). It is important to consider this diagno sis in patients who develop diarrhea in association with antibiotic use. Symptoms of pseudomembranous colitis may occur during or after antibiotic treatment and may range in severity from mild to life threatening. Mild cases usually respond to drug discontinuation alone. Moderate-to-severe cases should be managed with fluid, electrolyte, and protein supplementation. When the colitis is not relieved by drug discontin uance, or when it is severe, oral vancomycin is the drug of choice for antibiotic associated pseudomembranous colitis produced by $\mathrm{C}$ difficile. Other causes of colitis should be excluded.

\section{PRECAUTIONS}

General: Prolonged use may result in overgrowth of nonsusceptible organisms. If superinfection occurs, take appropriate measures.

Carefully monitor patients on dialysis. Adjust dosage of SUPRAX (cefixime) in patients with renal impairment and those undergoing continuous ambulatory peritoneal dialysis and hemodialysis. (See DOSAGE AND ADMINISTRATION.)

Prescribe cautiously in patients with a history of gastrointestinal disease, particularly colitis.

Drug interactions: No significant drug interactions have been reported to date Drug/Laboratory Test Interactions: A false-positive reaction for ketones in the urine may occur with tests using nitroprusside but not with those using nitroferricyanide.

SUPRAX administration may result in a false-positive reaction for glucose in the urine using Clinitest*", " Benedict's solution, or Fehling's solution. Use glucose tests based on enzymatic glucose oxidase reactions (such as Clinistix ${ }^{* *}$ or Tes-Tape ${ }^{* *}$

A false-positive direct Coombs test has been reported during treatment with other cephalosporin antibiotics; therefore, it should be recognized that a positive Coombs test may be due to the drug.

Carcinogenesis, Mutagenesis, Impairment of Fertility: Although no lifetime anima studies have been conducted to evaluate carcinogenic potential, no mutagenic potential of SUPRAX was found in standard laboratory tests. Reproductive studies revealed no fertility impairment in rats at doses up to 125 times the adult therapeutic dose. Usage in Pregnancy: Pregnancy Category B: Reproduction studies have been performed in mice and rats at doses up to 400 times the human dose and have revealed no evidence of harm to the fetus due to SUPRAX

There are no adequate and well-controlled studies in pregnant women. Because animal reproduction studies are not always predictive of human response, this drug should be used during pregnancy only if clearly needed.

Labor and Delivery: SUPRAX has not been studied for use during labor and delivery. Treatment should only be given if clearly needed.

Nursing Mothers: It is not known whether SUPRAX is excreted in human milk. Consider discontinuing nursing temporarily during treatment with this drug.

Pediatric Use: Safety and effectiveness of SUPRAX in children aged less than 6 months have not been established.

The incidence of gastrointestinal adverse reactions, including diarrhea and loose stools, in pediatric patients receiving the suspension, was comparable to adult patients receiving tablets

\section{ADVERSE REACTIONS}

Most adverse reactions observed in clinical trials were of a mild and transient nature. Less than four percent $(3.8 \%)$ of patients in the US trials discontinued therapy because of drug-related adverse reactions. Commonly seen adverse reactions in US trials of the tablet formulation were gastrointestinal events, which were reported in $30 \%$ of adult patients on either the bid or the qd regimen. Clinically mild gastrointestinal side effects occurred in $20 \%$ of all patients, moderate events occurred in $9 \%$ of all patients, and severe adverse reactions occurred in $2 \%$ of all patients. Individual event rates included diarrhea $16 \%$, loose or frequent stools $6 \%$, abdominal pain $3 \%$, nausea $7 \%$, dyspepsia $3 \%$, and flatulence $3 \%$. The incidence of gastrointestinal adverse reac tions, including diarrhea and loose stools, in pediatric patients receiving the suspension was comparable to adult patients receiving tablets.

Symptoms usually responded to symptomatic therapy or ceased when SUPRAX was discontinued.

Several patients developed severe diarrhea and/or documented pseudomembranous colitis, and a few required hospitalization.

The following adverse reactions have been reported following the use of SUPRAX Incidence rates were less than 1 in 50 (less than $2 \%$ ), except as noted above for gastrointestinal events.

Gastrointestinal: Diarrhea, loose stools, abdominal pain, dyspepsia, nausea, and vomiting. Several cases of documented pseudomembranous colitis were identified during the studies. The onset of pseudomembranous colitis symptoms may occur during or after therapy.

Hypersensitivity Reactions: Skin rashes, urticaria, drug fever, and pruritus. Erythema multiforme, Stevens-Johnson syndrome, and serum sickness have been reported rarely.

Hepatic: Transient elevations in SGPT, SGOT, and alkaline phosphatase.

Renal: Transient elevations in BUN or creatinine.

Central Nervous System: Headaches 3\%; dizziness.

Hemic and Lymphatic Systems: Transient thrombocytopenia, leukopenia, and eosinophilia. Prolongation in prothrombin time was seen rarely.

Other: Genital pruritus, vaginitis, candidiasis.

The following adverse reactions and altered laboratory tests have been reported for cephalosporin-class antibiotics:

Adverse Reactions: Allergic reactions including anaphylaxis, toxic epidermal necrolysis, superinfection, renal dysfunction, toxic nephropathy, hepatic dysfunction,

including cholestasis, aplastic anemia, hemolytic anemia, hemorrhage.

Several cephalosporins have been implicated in triggering seizures, particularly in patients with renal impairment when the dosage was not reduced (see DOSAGE AND ADMINISTRATION and OVERDOSAGE). If seizures associated with drug therapy occur, discontinue drug. Administer anticonvulsant therapy if clinically indicated.

Abnormal Laboratory Tests: Positive direct Coombs test, elevated bilirubin, elevated $L D H$, pancytopenia, neutropenia, agranulocytosis.

\section{OVERDOSAGE}

Gastric lavage may be indicated; otherwise, no specific antidote exists. Cefixime is not removed in significant quantities from the circulation by hemodialysis or peritonea dialysis. Adverse reactions in small numbers of healthy adult volunteers receiving single doses up to $2 \mathrm{~g}$ of SUPRAX did not differ from the profile seen in patients treated at the recommended doses.

- Clinitest and Clinistix are registered trademarks of Ames Division, Miles Laboratories, Inc. Tes-Tape is a registered trademark of Eli Lilly and Company.

LEDERLE LABORATORIES DIVISION

American Cyanamid Company, Pearl River, NY 10965

Under License of Fujisawa Pharmaceutical Co., Ltd., Osaka, Japan

Lederle Laboratories

A Division of American Cy
Wayne, New Jersey 07470

Cil Unoer ucensed Osaka, Japan

(c) 1990 Lederle Laboratories $7634-0$ 


\section{Before prescribing, please consult complete product information, a summary of which follows:}

WARNING: Bumex (bumetanide/Roche) is a potent diuretic which if given in excessive amounts, can lead to profound diuresis with water and electrolyte depletion. Therefore, careful medical supervision is required, and dose and dosage schedule have to be adjusted to the individual patient's needs. (See under DOSAGE AND ADMINISTRATION in complete product information.)

INDICATIONS AND USAGE: Edema associated with congestive heart failure, hepatic and renal disease, including the nephrotic syndrome.

Almost equal diuretic response occurs after oral and parenteral administration of Bumex. If Almost equal diuretic response occurs after oral and parenteral administration of Bumex. If impaired gastrointestinal absorption is suspected or oral adming

Successful treatment with Bumex following instances of allergic reactions to furosemide suggests a lack of cross-sensitivity.

CONTRAINDICATIONS: Anuria. Hypersensitivity and in patients in hepatic coma or in states of severe electrolyte depletion. Aithough Bumex can be used to induce diuresis in renal insufficiency. any marked increase in blood urea nitrogen or creatinine, of the development of oliguria during. therapy of patients with progressive renal disease, is an indication for discontinuation of treatment. WARNINGS: Dose should be adjusted to patient's needs. Excessive doses or too frequent administration can lead to profound water loss, electrolyte depletion, dehydration, reduction in blood volume and circulatory collapse with the possibility of vascular thrombosis and embolism, particularly in elderly patients.

Prevention of hypokalemia requires particular attention in patients receiving digitalis and diuretics for congestive heart failure, hepatic cirrhosis and ascites, states of aidosterone excess with norma renal function, potassium-losing nephropathy, certain diarrheal states, or other states where hypokalemia is thought to represent particular added risks to the patients.

in patients with hepatic cirihosis and ascites, sudden alleration in patients with hepatic cirmosis and ascites, sudden aiterations of electrolyte balance may precipitate hepatic encephalopathy and coma. Ireatment in such patients is best initiated in the hospital with small doses and careful monitoring of the patient's clinical status and electrolyte balance. Supplemental potassium and/or spironolactone may prevent hypokalemia and metabolic

In cats, dogs and guinea pigs. Bumex has been shown to produce ototoxicity. Since Bumex is abou 40 to 60 times as potent as furosemide, it is anticipated that blood levels necessary to produce ototoxicity will rarely be achieved. The potential for ototoxicity increases with intravenous therapy. especially at high doses.

Patients allergic to sulfonamides may show hypersensitivity to Bumex.

PRECAUTIONS: Measure serum potassium periodically and add potassium supplements or potassium-sparing diuretics, if necessary. Periodic determinations of other electrolytes are advised in patients treated with high doses or for prolonged periods, particularly in those on low salt diets. Hyperuricemia may occur. Reversible elevations of the BUN and creatinine may occur, especially Hyperuricemia may occur. Reversibie elevations of the BUN and creatinine may occur, especials
with dehydration and in patients with renal insufficiency. Bumex may increase urinary calcium with dehydration.

Possibility of effect on glucose metabolism exists. Periodic determinations of blood sugar should be done, particularly in patients with diabetes or suspected latent diabetes.

Patients should be observed regularly for possible occurrence of blood dyscrasias, liver damage or diosyncratic reactions.

Especially in presence of impaired renal function, use of parenterally administered Bumex should be avoided in patients to whom aminoglycoside antibiotics are also being given, except in lifethreatening conditions.

Drugs with nephrotoxic potential and bumetanide should not be administered simultaneously. Since lithium reduces renal clearance and adds a high risk of lithium toxicity, it should not be given with diuretics.

Probenecid should not be administered concurrently with Bumex.

Concurrent therapy with indomethacin not recommended.

Bumex may potentiate the effects of antihypertensive drugs, necessitating reduction in dosage. Interaction studies in humans have shown no effect on digoxin blood levels

Interaction studies in humans have shown Bumex to have no effect on warfarin metabolism or on plasma prothrombin activity.

Pregnancy: Bumex should be given to a pregnant woman only if the potential benefit justifies the potential risk to the fetus.

Bumetanide may be excreted in breast milk.

Pediatric use: Safety and effectiveness below age 18 not established.

ADVERSE REACTIONS: Muscle cramps, dizziness, hypotension, headache and nausea, and encephalopathy (in patients with preexisting liver disease)

Less frequent clinical adverse reactions are weakness, impaired hearing, rash, pruritus, hives, electrocardiogram changes, abdominal pain, arthritic pain, musculoskeletal pain and vomiting. Other clinical adverse reactions are vertigo, chest pain, ear discomfort, fatigue, dehydration. sweating, hyperventilation, dry mouth, upset stomach, renal failure, asterixis, itching, nipple tenderness, diarrhea, premature ejaculation and difficulty maintaining an erection.

Laboratory abnormalities reported are hyperuricemia, azotemia, hyperglycemia, increased serum creatinine, hypochloremia, hypokalemia, hyponatremia and variations in $\mathrm{CO}_{2}$ content, bicarbonate, phosphorus and calcium. Although manifestations of the pharmacologic action of Bumex, these conditions may become more pronounced by intensive therapy.

Also reported have been thrombocytopenia, deviations in hemoglobin, prothrombin time, hematocrit. WBC and differential counts. There have been rare spontaneous reports of thrombocytopenia from postmarketing experience.

Diuresis induced by Bumex may also rarely be accompanied by changes in LDH. total serum bilirubin, serum proteins, SGOT, SGPT, alkaline phosphatase, cholesterol, creatinine clearance. Increases in urinary glucose and urinary protein have also been seen.

DOSAGE AND ADMINISTRATION:

Oral Administration: The usual total daily dosage is 0.5 to $2.0 \mathrm{mg}$ and in most patients is given as a single dose.

Parenteral Administration: Administer to patients (IV or IM) with Gl absorption problem or who can not take oral. The usual initial dose is 0.5 to $1 \mathrm{mg}$ given over 1 to 2 minutes. If insufficient response, a second or third dose may be given at 2 to 3 hour intervals up to a maximum of $10 \mathrm{mg}$ a day HOW SUPPLIED: kablets, $0.5 \mathrm{mg}$ (light green), $1 \mathrm{mg}$ (yellow) and $2 \mathrm{mg}$ (peach), bottles of 100 and 500: Tel-E Dose ${ }^{5}$ cartons of 100. Imprint on tablets: $0.5 \mathrm{mg}-$ ROCHE BUMEX 0.5: $1 \mathrm{mg}$ - ROCHE BUMEX 1;2 mg - ROCHE BUMEX 2 Ampuls, $2 \mathrm{ml}, 0.25 \mathrm{mg} / \mathrm{ml}$, boxes of ten Vials, $2 \mathrm{ml}, 4 \mathrm{ml}$ and $10 \mathrm{mi}, 0.25 \mathrm{mg} / \mathrm{ml}$, boxes of ten.

Store all tablets, vials and ampuls at $59^{\circ}$ to $86^{\circ} \mathrm{F}$

\section{Roche Laboratories}

a division of Hoffmann-La Roche Inc

340 Kingsland Street, Nutley, New Jersey 07110-1199

We Wrote the Books on Patient Medication Education

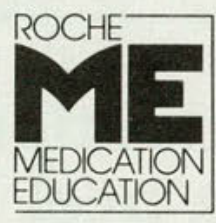

\section{Now we've written them in Spanish}

In 1978, Hoffmann-La Roche pioneered pharmaceutical patient information with the introduction of the Roche Medication Education (ME) program. We stand ready to meet the growing need for patient medication education in your practice. We invite you to take part in this valuable patient information program.

By simply checking the boxes below and mailing the coupon to Roche Medication Education, Roche Laboratories, a division of Hoffmann-La Roche Inc., Nutley, New Jersey 07110-1199, you may obtain a complimentary supply of the booklets providing specific information on Roche branded products listed below.

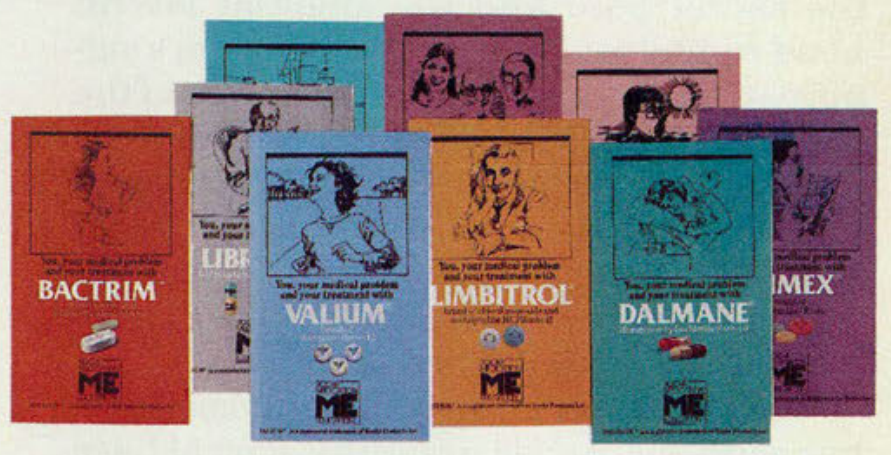

\section{Roche Product Books}

English Spanish version version

\begin{tabular}{|c|c|}
\hline . & $\begin{array}{l}\text { Bactrim }{ }^{T M} \text { (trimethoprim } \\
\text { and sulfamethoxazole/Roche) }\end{array}$ \\
\hline & Berocca \\
\hline & Bumex (bumetanide/Roche) ${ }^{*}$ \\
\hline & $\begin{array}{l}\text { Dalmane }{ }^{\star} \text { (brand of flurazepam } \\
\text { hydrochloride/Roche) CIV }\end{array}$ \\
\hline & Efudex (fluorouracil/Roche) \\
\hline & $\begin{array}{l}\text { Librium }^{\odot} \text { (brand of } \\
\text { chlordiazepoxide HCl/Roche) CIV }\end{array}$ \\
\hline & $\begin{array}{l}\text { Limbitrol }^{\circ} \text { (brand of chlordiazepoxide } \\
\text { and amitriptyline } \mathrm{HCl} / \text { Roche) CIV }\end{array}$ \\
\hline & Rocaltrol $^{\odot}$ (calcitriol/Roche) \\
\hline & Valium ${ }^{\circ}$ (brand of diazepam/Roche) CIV \\
\hline$\overline{N A M E}$ & \\
\hline
\end{tabular}

